\title{
First Evaluation of a Retinal Imaging Laser Eyewear System Based Low Vision Aid
}

This article was published in the following Dove Press journal:

Clinical Ophthalmology

\section{Mareile Stöhr \\ Dirk Dekowski \\ Nikolaos Bechrakis \\ Joachim Esser \\ Anja Eckstein \\ Michael Oeverhaus}

Department of Ophthalmology, University Hospital Essen, Essen, Germany
Correspondence: Mareile Stöhr Department of Ophthalmology, University Hospital Essen, Hufelandstr.

55, Essen 45147, Germany

Tel +49-20I-723-2976

Fax +49-20I-723-6755

Email Mareile.stoehr@uk-essen.de
Purpose: We conducted this trial to investigate a new wearable laser-eyewear (LEW). Images of an integrated camera are projected to the retina by a RGB-Laser $(<1 \mu \mathrm{W})$ and MEMS-mirror system. This enables a full-color live video as augmented reality embedded in the field of vision of the wearer. Thin parallel laser beams are projected following the principle of Maxwellian view through the center of the ocular lens to ensure independency of refractive errors. We performed a study with healthy subjects to test this independency.

Materials and Methods: LEW was tested in 20 healthy subjects (aged between 21 and 60 years) with hyperopia, myopia, astigmatism and/or presbyopia. Subjects underwent standardized visual acuity (VA) measurements (ETDRS) without any correction, with LEW and with best correction.

Results: We found no significant correlation between refraction and VA while using LEW in linear regression $(r=0.17)$. Still, younger participants performed better in terms of VA with the device compared to older participants despite no differences in BCVA $(p<0.01)$. The achievable VA with LEW was in general reduced compared to uncorrected VA ( 0.50 vs 0.40 $\log$ MAR). Only myopic subjects reached a significantly higher VA using LEW $(p<0.001)$. Presbyopic subjects showed enhanced near VA $(0.25 \operatorname{logMAR})$ by reading at $15 \mathrm{~cm}$ with LEW without any further necessary refractive correction. Nearly all patients $(80 \%)$ showed stereopsis without need for additional adjustments.

Conclusion: Our investigation could verify the independence of LEW of refractive errors. Therefore, the technology seems to be especially useful in patients with untreatable corneal conditions, e.g., after corneal burns, to achieve higher VA since the thin laser should penetrate even corneal opacities with less scattering.

Keywords: low vision, visual impairment; VI, corneal haze, visual rehabilitation, dystrophy, low vision aid; LVA, Maxwellian view, augmented reality; AR

\section{Synopsis}

A new portable retinal imaging laser-eyewear (LEW) enables direct projection of images of an integrated camera onto the retina, independent of refractive errors by using the Maxwellian view principle.

\section{Introduction}

Visual impairment (VI) is considered as a difficult condition worldwide both for the affected person and for the adequate care regarding therapy and adjustment of the best low vision aid (LVA) for the affected person. Age-related macular degeneration (AMD), diabetic retinopathy and glaucoma are in the majority of cases the reason for VI and blindness in developed countries. ${ }^{1}$ Other diseases affecting the anterior 
segment of the eye can also leading to blindness. These includes VI due to corneal diseases, e.g., keratokonus, corneal dystrophies, opacities caused by chemical or thermal burns and graft-versus-host disease (GvHD). While the most patients receive effectively treatment with surgery, like keratoplasty or limbal-stem-cell transplantation, some patients cannot undergo surgery due to ocular risk factors (e.g., uveitis, uncontrolled IOP, vascularization, etc. $)^{2,3}$ or due to other comorbidities or even rejection of surgery. Often, there are long waiting periods of months or years for patients who could benefit from surgery because a suitable graft is necessary for them. ${ }^{4}$ These patient cohorts have to sustain from VI and from its implications regarding social miscommunication, reading disability, problems in recognizing faces and in accomplishing activities of daily living. Higher prevalence of depression, decrease of self-sufficiency and a loss of quality of life are the consequences of the above mentioned problems of patients suffering from VI. ${ }^{5-7}$ Therefore, it is important to support the patient's visual rehabilitation and subsequently to increase their autonomy. Reading ability can be successfully improved by LVAs, e.g., electronic video (CCTV) or optical magnifiers. ${ }^{8}$ For most of visually impaired patients, magnification is currently the only option to improve their vision. However, patients with impairment of the optic media and preserved sensory function could enhance their vision by bypassing the diseased anterior part of the eye. ${ }^{3}$

A new type of retinal imaging LEW might be able to deliver such a bypass: The new technique allows projecting images directly onto the retina and improving thereby the VA of patients. Firstly, this was tried using a technique similar to the "Scanning Laser Ophthalmoscope" (SLO), which was used to project Landolt C's onto the retina in 1981. ${ }^{9}$ Later Furness et al investigated the possibility of a direct retinal projection by means of a low-energy laser for LVAs. ${ }^{10}$ By using a laser, sharp images could be projected onto the retina, independent of the patient's refractive errors and focusing ability. ${ }^{11}$ This could be achieved by the principle of "Maxwellian view," in which the thin parallel laser beam projects images through the center of the ocular lens directly onto the retina. ${ }^{12}$ This laser projection technique allowed also passing through corneal opacities. The image was projected in a raster pattern, pixel by pixel onto the retina by means of a red laser diode and a mechanical resonance scanner (MRS). ${ }^{13}$ As a result of advances in recent years, technology has been further developed to project full color images of a camera, integrated into a spectacle, directly onto the retina. ${ }^{12,14,15}$ For this Retinal Imaging Laser Eye Wear (RILEW), a RGB (Red, Green and Blue)-Laser was used instead of the red laser diode by Furness et al, and the MRS-mirror was replaced by a microelectromechanical (MEMS) system, which allows even higher scan speeds (about 20ns) and thus higherresolution (see Figure 1). Due to an autofocus and automated contrast settings the device can be operated in various circumstances and lighting conditions. The projection eyewear offers the unique opportunity to improve the VA by direct retinal projection in patients with corneal diseases. The wearer sees a full-color video, in real time, monocular as an augmented reality (AR) embed in his central field of vision, which still allows for peripheral vision opposed to virtual reality (VR) systems. Full color is realized by using a Red $(640 \mu \mathrm{m}$ wavelength), Green $(515 \mu \mathrm{m})$ and Blue $(465 \mu \mathrm{m})$ laser diode. ${ }^{12}$

To be used as a LVA a laser eyewear system must satisfy two conditions: First, it is of extreme importance that the device inflicts no harm to the eye. Secondly, it should still be able to project clear images to be useful as a LVA.

The laser used in our study device is classified as a Class1-laser according the IEC60825-1. The low energy output could be shown to be lower compared to a fluorescent lamp and is therefore harmless to the eye. ${ }^{12,16}$ To evaluate the second condition, if the device is, despite the low energy output, still able to project clear images independent of

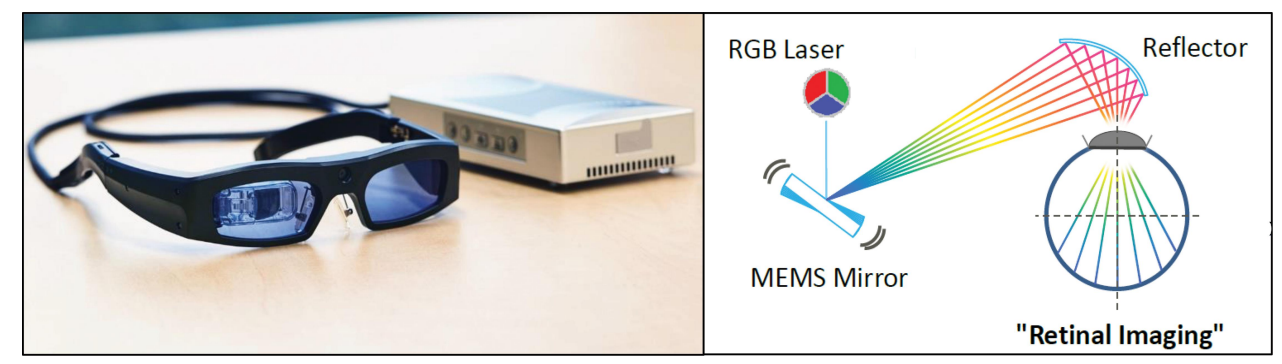

Figure I The retinal imaging laser eyewear projects the images of the integrated camera directly onto the retina of one eye via a RGB-Laser and a MEMS mirror and reflector system. Copyright by QD Laser Inc. Permission for publication was granted. 
refractive errors we performed this prospective, single-site, clinical trial in healthy subjects. Secondary objectives were to investigate if the wearer experiences double vision while using the LEW, if reading is possible and if magnification by approximation is feasible.

\section{Materials and Methods Study Cohort}

In this clinical investigation subjects were recruited by being briefed with brochures at University Hospital Essen about implementation of this trial. Subjects $(n=20)$ were eligible for the study meeting the following criteria: Refractive errors between -7 to +6 Diopters (Dpt) (myopia, hyperopia, astigmatism and/or presbyopia) but no limitation in visual ability due to any eye disease (e.g. glaucoma, AMD, diabetic retinopathy etc), best corrected visual acuity (BCVA) $\geq 0.8$ (20/25 Snellen), ability to read, write and communicate fluently in German, aged between 18 and 60 years.

\section{Ophthalmological Investigation}

Subjects were examined in a standardized orthoptic and ophthalmological examination of the right and left eye using Titmus und Lang test, slit-lamp microscopy and fundoscopy to exclude ocular diseases. Additionally, all subjects received a measurement of laser-interference visual acuity (LIVA), objective and subjective refraction measurements of both eyes, as well as an amplitude of fusion measurement. Refraction was performed according the Early Treatment of Diabetic Retinopathy StudS (ETDRS) protocol in $2 \mathrm{~m}$ distance using Chart "R". Determining the best-corrected visual acuity (BCVA) the least negative or most positive spherical lens was used, additionally the least negative cylindrical lens that lead to the best visual acuity (VA) of each subject. To secure consistency and reproducibility, all examinations were performed by the same investigator.

\section{Visual Acuity Measurements}

Following enrollment, patients were thoroughly instructed about the LEW and its usage. All subsequent standardized VA measurements were initially performed without any correction, following with LEW (RETISSA Model ES2, QD Laser Inc., Japan, Tokyo) and finally tested with best correction. VA was measured both in the far $(2 \mathrm{~m})$ and in the near $(40 \mathrm{~cm})$ distance following the ETDRS protocol. The eyes were examined separately (right eye first) at both distances. Illuminated Bailey Lovie "Chart 1" was used for the right eye and "Chart 2" for the left eye. In the visual test using the LEW, the occlusion of both glasses was used to determine the VA exclusively via the retinal projection. To perform under same condition all VA measurement were accomplished in the same darkened room. Using the method described by Ferris, all correctly read letters were counted. ${ }^{17,18}$ Determining the differences of VA, the results are shown in logMAR units.

\section{Reading Speed}

Reading speed was assessed using the International Reading Speed Texts (IReST) to evaluate the reading speed with LEW in comparison to the reading speed with best correction of subjects. The different texts consist of the same length, the same linguistic complexity and the same degree of difficulty to secure comparability between the different texts. Reading speed was first performed using LEW. Afterwards, the reading speed with best correction was performed with a different text of IReST matched to the same difficulty level. Needed time and misread words were assessed while the patients read aloud. The reading speed was calculated using the formula [(correctly read words/reading time $) \times 60]$. The reading speed was given in words per minute (wpm) and was interindividually comparable to the normative reading speed provided by the IreST-study-group. ${ }^{19,20}$

\section{Influence on Stereopsis}

After wearing the LEW for the first time, subjects were asked about double images in near and distance vision. If any double images occurred, different adjustments were made stepwise until the patient had no longer double vision: First, the pupil distance was adjusted, then best correction added and at last prisms applied.

\section{Statistical Evaluation}

All calculations were performed using Excel 2019 (Microsoft Corporation, Version 16.0 for Windows) and GraphPad PRISM 8 for Windows (Software Inc., San Diego, CA, USA). Median values and mean and standard deviation $( \pm \mathrm{SD})$ for the different metric data were assessed. If Pearson's test displayed normal distribution, differences between the groups were calculated with twotailed Student's $t$-test. Otherwise, Mann-Whitney-U-Test was used. Evaluation of group distribution of binary variables followed Fisher's exact test. Testing the association between VA and refraction, linear regressions have been calculated. To assess further influential factors, multiple 
linear regressions have been performed. Level of statistical significance was defined two-tailed $(2 \alpha<0.05)$. P-values are given with $\alpha$-adjustment for multiple testing.

\section{Results}

\section{Study Cohort}

Recruitment period was between 10th November 2017 and 19th January 2018. Twenty subjects were recruited and eligible for participating in this clinical study. The mean age of the study population was $41.2 \pm 14$ (range: $21-60$ ). All patients had refractive errors in accordance with the inclusion criteria (range: +4.5 till $-6.5 \mathrm{dpt}$, see Table 1 and Figure 2). The study population consisted of few younger subjects with myopia (mean age: 34.6 ) and more older subjects with hyperopia (mean age: 46.6).

\section{Ophthalmological Investigations}

All 20 patients were eligible according the inclusion and exclusion criteria and had no ocular diseases besides refractive errors. Examination with LIVA and fundoscopy assured, that subjects had no concomitant diseases, which could limit the retinal projection. In the final analysis a total of 40 eyes $(100 \%)$ were included.

\section{Visual Acuity Assessments}

The whole study cohort showed normal values for BCVA according to the inclusion criteria. No differences occurred between myopic and hyperopic patients. Mean VA with LEW was $0.50 \pm 0.08 \log$ MAR [range: $0.64-0.28$ ). We found no significant correlation between refraction and VA while using LEW in linear regression $(p=0.25$, $\mathrm{r}=0.17$, s. Figure $3 \mathrm{~A}$ ). Younger participants could achieve a higher VA with LEW in comparison to older participants $(p<0.01)$ despite no differences in BCVA. To further explore this we also performed a linear regression for age, which showed a significant correlation $(\mathrm{p}=0.007$, $\mathrm{r}=0.47$, see Figure $3 \mathrm{~B}$ ). Uncorrected VA showed an expected correlation with the amount of myopia in contrast to the VA with LEW (Figure 3C). As expected, such correlation was not present in the hyperopic patients due to their accommodation possibility. Magnification with LEW by digital zoom resulted in an improvement of $0.21 \operatorname{logMAR}$, which is equal to 2 lines (see Figure 4). Evaluation of near VA showed that presbyopic subjects $(n=5)$ could increase their near VA (up to $0.25 \log$ MAR) by reading at a shorter distance $(15 \mathrm{~cm})$ using LEW without any further correction.

\section{Reading Speed}

The reading speed test was performed with LEW and with best correction. Initially, reading was assessed with LEW and finally with best correction. Reading was for all patients possible, but with LEW (mean: $76.1 \mathrm{wpm}$ ) significantly slower compared to best correction (mean: 174.2 wpm) (see Figure 5).

\section{Influence on Stereopsis}

Eighty percent of the subjects received stereopsis without need for additional corrections. In the minority subjects, fusion could be reached with either best correction (10\%), additional use of prisms (5\%) or adjustment of pupillary distance $(5 \%)$. All these patients showed heterophoria in the orthoptic examination and a reduced amplitude of fusion.

\section{Discussion}

In this investigator-initiated, prospective clinical study a detailed evaluation of patient's VA while wearing glasses with a new laser technology could be successfully assessed. The independency of refractive errors while using a retinal imaging system following the principle of Maxwellian view could be shown. Thus, the potential of LEW should be tested in patients with corneal diseases who cannot be sufficiently treated with visual aids or surgery.

\section{Independency of Refractive Errors}

In this clinical trial we could confirm that VA with retinal projection is independent of refractive errors. The theoretical independency described by Sugawara et al could be confirmed. ${ }^{21}$ Today, there is no comparable device working with the principle of Maxwellian view. Previous trials by Furness et al which used also a retinal projection system did not analyze the VA with LEW, but only the concept and safety. In our trial the mean VA with LEW was $0.50 \log$ MAR, which is comparable to an unpublished trial in Japan performed to investigate the device's influence on the VA in healthy adults and cataract patients. ${ }^{22}$ Whereas best corrected VA and LEW were not significantly affected by the amount of refractive error, uncorrected VA deteriorated with increasing myopia. To perform under same conditions VA testing was accomplished in the same darkened room. Since the experience of $\mathrm{AR}$ is dependent on the ambient contrast ratio further tests for the suitability for daily use and comparison of different light ratios are necessary. ${ }^{23} \mathrm{~A}$ significant 
Table I Patient Characteristics: Refractive Errors and VA (LE=left Eye, RE= Right Eye, UCVA= Uncorrected Visual Acuity, BCVA= Best Corrected Visual Acuity, LEW=Laser-Eyewear)

\begin{tabular}{|c|c|c|c|c|c|c|c|}
\hline \multirow[t]{2}{*}{ Subject } & \multirow[t]{2}{*}{ Age } & \multicolumn{3}{|c|}{ Refractive Errors } & \multicolumn{3}{|c|}{$\begin{array}{l}\text { Visual Acuity Measurement } \\
\text { (logMAR) }\end{array}$} \\
\hline & & Sph & cyl & A & UCVA & BCVA & LEW \\
\hline I-RE & 37 & -1.50 & -0.25 & 97 & 0,26 & $-0,16$ & 0,44 \\
\hline 2-RE & 25 & 4.25 & -0.50 & 167 & $-0,16$ & $-0,10$ & 0,44 \\
\hline $3-R E$ & 52 & -1.25 & -0.50 & 82 & 0,48 & 0,12 & 0,60 \\
\hline 4-RE & 24 & -2.50 & -0.25 & 119 & 0,64 & $-0,12$ & 0,54 \\
\hline $5-R E$ & 21 & -3.00 & -0.50 & 1 & 0,90 & $-0,22$ & 0,56 \\
\hline 6-RE & 51 & 0.75 & -0.25 & 41 & 0,12 & $-0,16$ & 0,52 \\
\hline 7-RE & 24 & -6.50 & -1.00 & 79 & 0,74 & $-0,10$ & 0,38 \\
\hline 8-RE & 58 & 1.50 & -0.50 & 43 & 0,50 & 0,06 & 0,64 \\
\hline 9-RE & 53 & 1.00 & -0.25 & 39 & 0,24 & $-0,10$ & 0,64 \\
\hline I0-RE & 28 & -3.75 & -0.50 & 165 & 0,64 & $-0,20$ & 0,44 \\
\hline II-RE & 26 & -1.25 & -0.75 & 110 & 0,36 & $-0,20$ & 0,46 \\
\hline I2-RE & 54 & 1.00 & -1.50 & 180 & 0,10 & $-0,14$ & 0,54 \\
\hline I3-RE & 60 & 1.50 & -0.50 & 174 & 0,42 & $-0,12$ & 0,56 \\
\hline I4-RE & 24 & 4.50 & -1.00 & $|7|$ & 0,04 & $-0,22$ & 0,48 \\
\hline I5-RE & 28 & 4.75 & -1.50 & 100 & 0,08 & $-0,20$ & 0,46 \\
\hline I6-RE & 51 & 0.50 & -2.50 & 180 & 0,32 & 0,00 & 0,48 \\
\hline I7-RE & 54 & 2.50 & -0.50 & 116 & 0,50 & $-0,04$ & 0,44 \\
\hline I8-RE & 46 & -1.25 & 0.00 & 180 & 0,50 & $-0,06$ & 0,54 \\
\hline 19-RE & 53 & -4.00 & -1.25 & 100 & $\mathrm{I}, 04$ & $-0,10$ & 0,52 \\
\hline 20-RE & 54 & 2.25 & -1.00 & 26 & 0,22 & 0,02 & 0,60 \\
\hline I-LE & 37 & -1.75 & -0.25 & 155 & 0,42 & $-0,14$ & 0,26 \\
\hline 2-LE & 25 & 4.00 & -1.00 & 170 & 0,12 & $-0,16$ & 0,34 \\
\hline 3-LE & 52 & -1.00 & -0.50 & 93 & 0,32 & 0,02 & 0,30 \\
\hline 4-LE & 24 & -2.50 & -0.50 & 42 & 0,62 & $-0,20$ & 0,24 \\
\hline 5-LE & 21 & -1.50 & -1.50 & 174 & 0,22 & $-0,10$ & 0,36 \\
\hline 6-LE & 51 & 0.75 & -0.25 & 164 & 0,14 & $-0,18$ & 0,32 \\
\hline 7-LE & 24 & -7.00 & -0.75 & 121 & 0,52 & $-0,14$ & 0,10 \\
\hline 8-LE & 58 & 1.25 & 0.00 & 180 & 0,40 & 0,14 & 0,36 \\
\hline 9-LE & 53 & 0.50 & 0.00 & 180 & 0,10 & $-0,14$ & 0,36 \\
\hline I0-LE & 28 & -2.75 & -0.50 & 7 & 0,54 & $-0,18$ & 0,24 \\
\hline II-LE & 26 & -0.75 & -0.75 & 85 & 0,10 & $-0,20$ & 0,26 \\
\hline 12-LE & 54 & 0.75 & 0.00 & 173 & $-0,04$ & $-0,14$ & 0,30 \\
\hline I3-LE & 60 & 1.75 & -0.50 & 151 & 0,38 & $-0,22$ & 0,34 \\
\hline I4-LE & 24 & 4.25 & -0.50 & 179 & 0,06 & 0,02 & 0,34 \\
\hline I5-LE & 28 & 5.25 & -3.00 & 20 & 0,30 & $-0,20$ & 0,20 \\
\hline I6-LE & 51 & 0.00 & -2.25 & 172 & 0,56 & 0,04 & 0,26 \\
\hline I7-LE & 54 & 2.75 & 1.25 & 7 & 0,48 & $-0,10$ & 0,30 \\
\hline I8-LE & 46 & -2.25 & 0.00 & 180 & 0,46 & $-0,10$ & 0,30 \\
\hline 19-LE & 53 & -4.00 & -0.50 & 168 & 0,96 & $-0,10$ & 0,32 \\
\hline 20-LE & 54 & 3.00 & 1.00 & 165 & 0,62 & 0,02 & 0,50 \\
\hline
\end{tabular}

correlation between age and VA with LEW was shown in linear regression, which is in accordance with previous trials which could show that VA generally decreases with age even in healthy individuals. ${ }^{24}$ Due to the independency of refraction, presbyopic subjects could increase their near VA reading in a shorter distance of $15 \mathrm{~cm}$ using $L E W$ without need for any further spherical lens adjustment and myopic subjects could improve their distance VA. Though these refractive errors are currently better corrected with glasses, patients with irregular astigmatism and keratoconus might already profit from LEW, although the maximum VA with LEW is still lower as with customized 


\section{Spherical refraction errors}

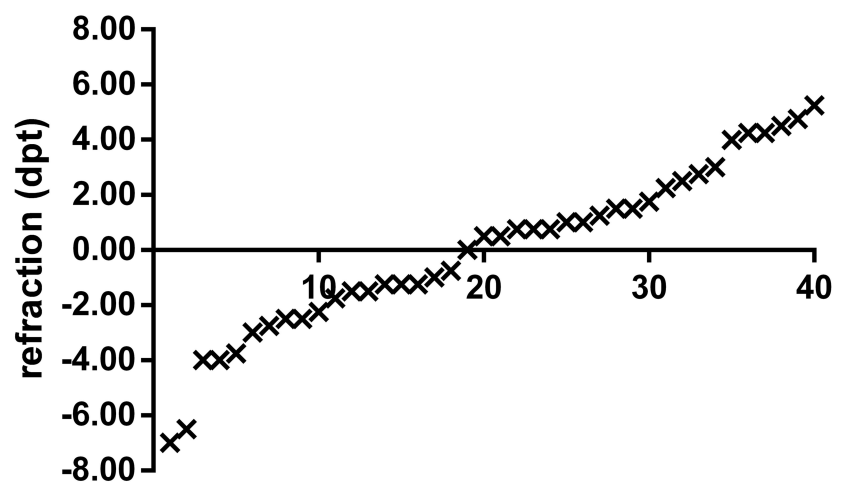

Figure 2 Distribution of the spherical refraction values of the 20 study participants (40 eyes) in Diopters (dpt).

contact lenses. However, some patients cannot tolerate contact lenses or have immense difficulties with the handling. Furthermore, future advances in the technology already promise VA up to $20 / 20$ by improving the image resolution with a thinner laser beam. ${ }^{16}$ The current system could now already be beneficial for patients who suffer

A
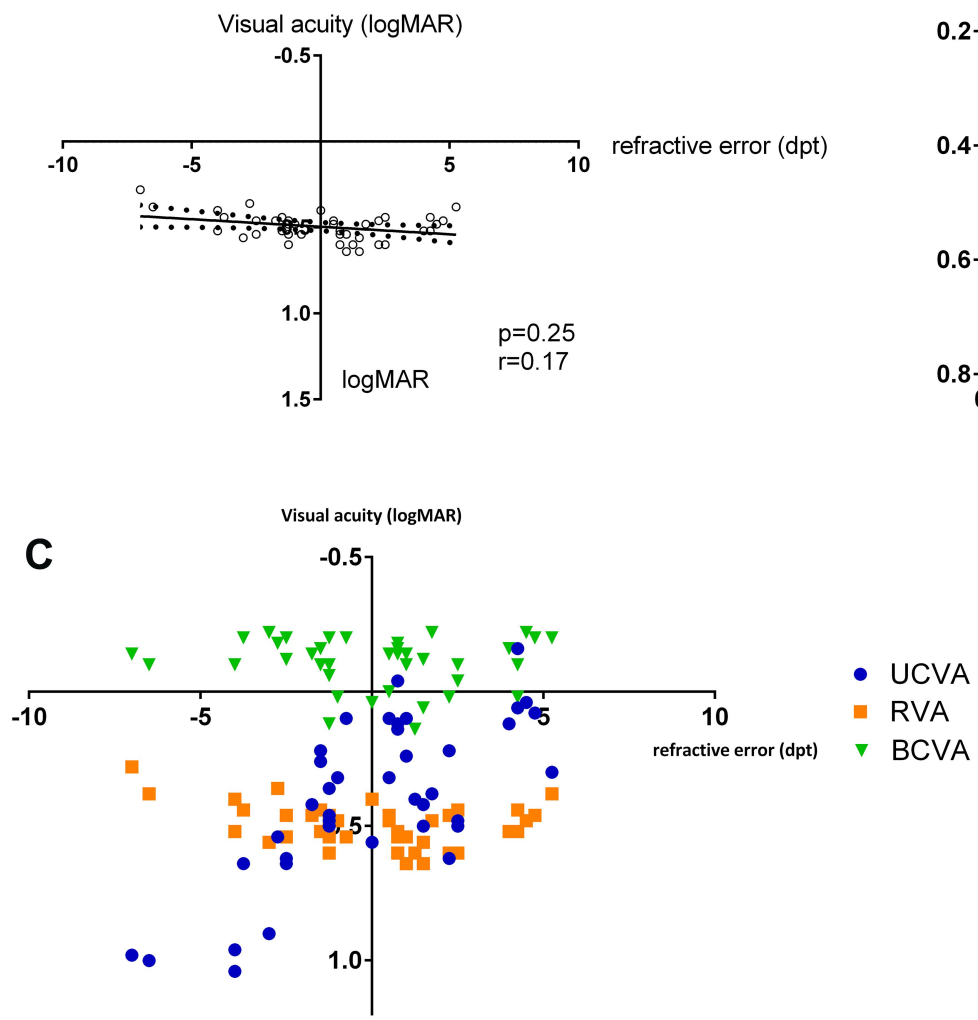

under corneal diseases as dystrophies, GvHD and scars after chemical burns, since it is expected to pass through corneal opacities and is not affected by irregular corneal surfaces. These patients are more difficult to treat with LVAs and should show an increase in their VA by using LEW instead of only making best use of their remaining

Visual acuity (logMAR)

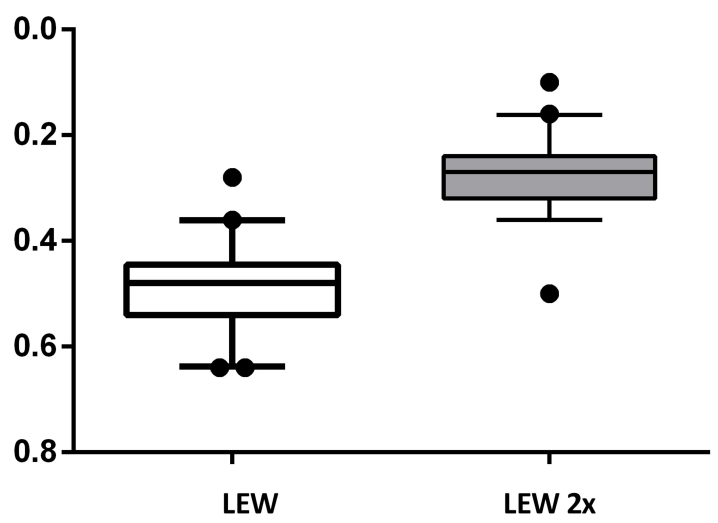

Figure 4 VA with LEW (white) and with LEW and 2-fold magnification (gray)

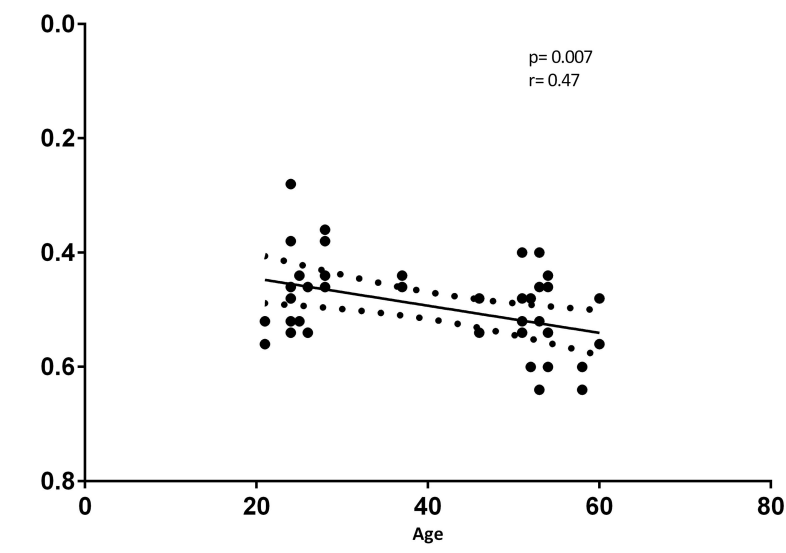

Figure 3 (A) Linear regression between VA with LEW and refraction showed no correlation, (B) Linear regression between VA with LEW and age showed a significant correlation. Higher age was associated with lower VA. (C) VA with LEW (orange), uncorrected (UCVA, blue) and best correction (BCVA, green). 


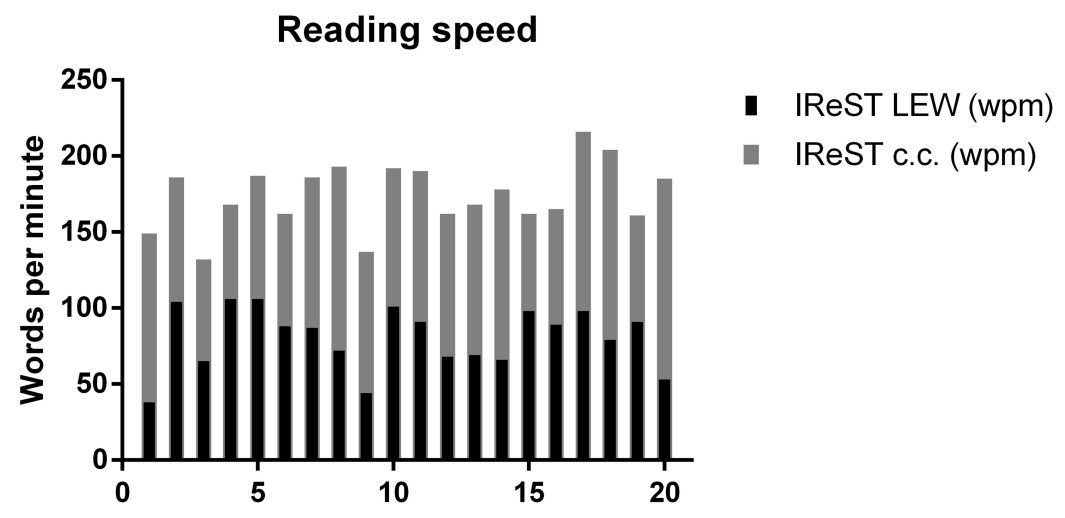

Figure 5 Reading speed (words per minute) with LEW (black) and best correction (gray).

vision by magnification. ${ }^{3}$ This has been recently evaluated in a clinical trial (DRKS00016583), which results will be published soon. Further improvements of retinal scanning displays might miniaturize the design which will further improve the mobility of such an LVA. ${ }^{25,26}$

\section{Reading}

Since the LEW is designed for low vision patients we also analyzed the reading ability with the device. Reading with LEW was possible, but slower compared to best correction. This can be explained by the limited visual field and VA. Training and the magnification feature might increase reading speed with LEW. In the future, an eye tracking feature might enable the device to maintain retinal projection besides eye movements. Overall, it will presumably be easier for the visual impaired patient to read short texts, e.g., price tags than books. However, reading was only tested at normal reading distance of $33 \mathrm{~cm}$. Due to the property of the LEW to function independently of the refraction, the LEW can used in shorter reading distances even in older patients. Like this the principle of magnification by approximation can benefit the patients, which is otherwise only possible in small children with high accommodation. ${ }^{27}$

\section{Binocular Single Vision}

As an example of the functioning of human perception and how it can be manipulated by an AR system, we considered an important phenomenon: the stereopsis. Since, the wearer sees two separate images of reality the visual perception system needs to fuse the two images to avoid diplopia. In our trial, nearly all subjects could achieve stereopsis without any additional adjustments. The eyes adjusted as in normal viewing to focus objects onto the fovea and fused the images. The subjects who experienced diplopia had all slight heterophoria. Here, fusion while wearing LEW could be achieved with best correction, pupillary distance adjustment or prisms. The LEW system provides horizontal view angle of approximately $26^{\circ}$, displayed on the retina augmented in the monocular field of view of $160^{\circ}$ horizontal and $130^{\circ}$ vertical. This means that the user loses the image when the eyeball is rotating and the position of the eyes could influence the stereopsis test. Therefore, a straight position of the viewer 's eyes is important and the pupillary distance adjustment was the first adjustment that could reduce slighty diplopia. Future technical improvement might minimize this problem by including on-axis gaze tracking. ${ }^{15}$ Already in 1858 Panum described the fusion ability despite small disparities between two separate images. ${ }^{28} \mathrm{He}$ evaluated the tolerance areas regarding the retinal location and could show that the amount of fused disparity is dependent on the image quality and the eccentricity of the retinal location. This and the knowledge that especially peripheral vision is responsible for stereopsis, could explain why all subjects were able to fuse the AR image with their second eye. ${ }^{29,30}$ Since only the central retinal area receives a virtual image and the peripheral retinal areas of both eyes see the same picture of the environment and the fusion is mediated anyway by peripheral retinal areas, ${ }^{31}$ the LEW does not appear to represent a major problem for binocular vision. This is gratifying, since VR systems often experience discomfort due to the perception of virtual images for both eyes. ${ }^{32}$ Future investigations might further evaluate the stereoscopic vision and the ability to perform tasks of daily living as grabbing objects.

\section{Limitation}

In this study, no adverse events occurred when wearing the laser eyewear; for example, symptoms such as impaired perception, headache or nausea, which occur in VR systems. ${ }^{32}$ However, the laser eyewear was worn for more 
a maximum of 30 minutes in total. Long-term observations are necessary for further statements regarding the safety. VA was assessed when LEW was used for the first time. Learning or training could lead to an improvement. Considering that the projection glasses could be useful for low vision patients in the future, the trial presents the foundation for the development of a novel LVA. The latest study did not include any orientation or mobility training, but this is relevant in the patient's point of view when using LEW as a visual aid.

\section{Conclusion}

In this clinical trial we evaluated the independency of refractive errors by using the LEW's retinal projection. While wearing LEW all subjects could accomplish stereoscopic vision. Only in minor cases of slight heterophoria were further corrections needed to achieve stereopsis. While using LEW, lens and cornea are not required for focusing. Therefore, especially patients with VI due to untreatable corneal diseases might benefit from the novel the retinal projection technology. Since the retinal projection circumvent their cause of impairment and scan the image directly onto the healthy retina, an increase of the patient's VA is possible. In summary, the LEW projection glasses are equipped with a new technology that in the future may offer a new possibility for visually impaired patients to enhance their visual function and the associated quality of life. Further studies are needed to address this topic.

\section{Abbreviations}

AMD, age-related macular degeneration; BCVA, best corrected visual acuity; CCTV, closed-circuit television; GvHD, Graft versus host disease; IOP, intraocular pressure; IReST, International Reading Speed Texts; MEMS, microelectromechanical system; LEW, laser-eyewear; LIVA, laser interference visual acuity; LVA, low vision aid; VA, visual acuity; VI, visual impairment.

\section{Data Sharing Statement}

The authors will provide the deidentified data on reasonable request via Zenodo clinical trial platform under this link: https://doi.org/10.5281/zenodo.4067996.

\section{Ethics Statement}

The Ethics Committee of the University of DuisburgEssen approved the conduct of this clinical study on healthy volunteers on 24 August 2017 (approval number: 17-7537-BO). In compliance with the Declaration of
Helsinki, written informed consent was obtained from all trial participants. The clinical study was listed in the DRKS-German Clinical Trials Register (DRKS00013300).

\section{Acknowledgments}

This study was supported by the Junior Clinician Scientist program of the University Medicine Essen Clinician Scientist Academy (UMEA) and the Open Access Publication Fund of the University of Duisburg-Essen.

\section{Disclosure}

M. Stöhr, D. Dekowksi, N. Bechrakis, J. Esser and M. Oeverhaus state that they have no conflicts of interest. A. Eckstein received funds as medical advisor for QD Laser Inc, reports grants from QD Laser outside the submitted work, and reports no other potential conflicts of interest for this work.

\section{References}

1. Knauer C, Pfeiffer N. [Blindness in Germany-today and in 2030]. Der Ophthalmologe. 2006;103(9):735- 741. doi:10.1007/s00347006-1411-y.German.

2. Nguyen NX, Seitz B, Martus P, Langenbucher A, Cursiefen C. Longterm topical steroid treatment improves graft survival following normal-risk penetrating keratoplasty. Am J Ophthalmol. 2007;144 (2):318-319. doi:10.1016/j.ajo.2007.03.028

3. Oeverhaus M, Stöhr M, Hirche H, Esser J, Eckstein A. Visual rehabilitation of patients with corneal diseases. BMC Ophthalmol. 2020;20. doi:10.1186/s12886-020-01436-7

4. Pauklin M, Steuhl KP, Meller D. [Limbal stem cell deficiency after chemical burns: investigations on the epithelial phenotype and inflammation status]. Der Ophthalmologe. 2009;106(11):995-998. doi:10.1007/s00347-008-1898-5.German.

5. Brody BL, Gamst AC, Williams RA, et al. Depression, visual acuity, comorbidity, and disability associated with age-related macular degeneration. Ophthalmology. 2001;108(10):1893-1900; discussion 1900-1891.

6. Trousdale ER, Hodge DO, Baratz KH, Maguire LJ, Bourne WM, Patel SV. Vision-related quality of life before and after keratoplasty for Fuchs' endothelial dystrophy. Ophthalmology. 2014;121 (11):2147-2152. doi:10.1016/j.ophtha.2014.04.046

7. Salive ME, Guralnik J, Glynn RJ, Christen W, Wallace RB, Ostfeld AM. Association of visual impairment with mobility and physical function. J Am Geriatr Soc. 1994;42(3):287-292. doi:10. 1111/j.1532-5415.1994.tb01753.x

8. Nguyen NX, Weismann M, Trauzettel-Klosinski S. Improvement of reading speed after providing of low vision aids in patients with age-related macular degeneration. Acta Ophthalmol. 2009;87 (8):849-853. doi:10.1111/j.1755-3768.2008.01423.x

9. Mogilnicka EM, Webb M. Comparative studies on the distribution of gold, copper and zinc in the livers and kidneys of rats and hamsters after treatment with sodium [195Au]-aurothiomalate. J Applied Toxicology. 1981;1(6):287-291.

10. Viirre E, Johnston R, Pryor H, Nagata S, Furness TA 3rd. Laser safety analysis of a retinal scanning display system. J Laser Appl. 1997;9(5):253-260. doi:10.2351/1.4745467 
11. Viirre E, Pryor H, Nagata S, Furness TA 3rd. The virtual retinal display: a new technology for virtual reality and augmented vision in medicine. Stud Health Technol Inform. 1998;50:252-257.

12. Sugawara M, Suzuki M, Miyauchi N. 14-5L: late-News Paper: retinal Imaging Laser Eyewear with Focus-Free and Augmented Reality. SID Symposium Digest Technical Papers. 2016;47(1):164-167.

13. Kleweno CP, Seibel EJ, Viirre ES, Kelly JP, Furness TA 3rd. The virtual retinal display as a low-vision computer interface: a pilot study. J Rehabil Res Dev. 2001;38(4):431-442.

14. Iyama C, Shigeno Y, Hirano E, et al. QD laser eyewear as a visual field aid in a visual field defect model. Sci Rep. 2019;9(1):1010. doi:10.1038/s41598-018-37744-8

15. Zhan T, Yin K, Xiong J, He Z, Wu ST. Augmented reality and virtual reality displays: perspectives and challenges. iScience. 2020;23 (8):101397. doi:10.1016/j.isci.2020.101397

16. Sugawara M, Suzuki M, Miyauchi N Image quality of retinal projection laser eyewear: how to achieve high resolution and free focus in proper balance. Yokohama, Japan: The 6th Laser Display and Lighting Conference 2017 (LDC 17). 2017.

17. Ferris FL 3rd, Kassoff A, Bresnick GH, Bailey I. New visual acuity charts for clinical research. Am J Ophthalmol. 1982;94(1):91-96. doi:10.1016/0002-9394(82)90197-0

18. Bailey IL, Lovie JE. The design and use of a new near-vision chart. Am J Optom Physiol Opt. 1980;57(6):378-387. doi:10.1097/ 00006324-198006000-00011

19. Hahn GA, Penka D, Gehrlich C, et al. New standardised texts for assessing reading performance in four European languages. $\mathrm{Br}$ J Ophthalmol. 2006;90(4):480-484.

20. Trauzettel-Klosinski S, Dietz K, Group IRS. Standardized assessment of reading performance: the new international reading speed texts IReST. Invest Ophthalmol Vis Sci. 2012;53(9):5452-5461. doi:10. 1167/iovs.11-8284

21. M SM S, Miyauchi N. Retinal imaging laser eye wear with focus-free and augmented reality. Sid Symposium Digest Technical Papers. 2016;47(1):164-167. doi:10.1002/sdtp.10626
22. Yamauchi T, Sugawara M, Suzuki M, et al. Visual function in healthy adults and cataract patients wearing retina scanning laser eyewear. In Preparation. 2019.

23. Lee J, Zhan T, Wu S-T. Prospects and challenges in augmented reality displays. Virtual Reality Intelligent Hardware. 2019;1:77.

24. Martinez-Roda JA, Vilaseca M, Ondategui JC, Aguirre M, Pujol J. Effects of aging on optical quality and visual function. Clin Exp Optom. 2016;99(6):518-525. doi:10.1111/cxo.12369

25. Mi L, Chen CP, Lu Y, Zhang W, Chen J, Maitlo N. Design of lensless retinal scanning display with diffractive optical element. Opt Express. 2019;27(15):20493-20507. doi:10.1364/OE.27.020493

26. Chen J, Mi L, Chen CP, Liu H, Jiang J, Zhang W. Design of foveated contact lens display for augmented reality. Opt Express. 2019;27 (26):38204-38219. doi:10.1364/OE.381200

27. Barker L, Thomas R, Rubin G, Dahlmann-Noor A. Optical reading aids for children and young people with low vision. Cochrane Database Systematic Reviews. 2015;(3):Cd010987.

28. Panum PL. [Physiological Evalutions of Binocular Vision]. 1. Kiel. Schwersche Buchhandlung;1858. German.

29. Burian HM. Fusional movements - Role of peripheral retinal stimuli. Arch Ophthalmol. 1939;21(3):486-491. doi:10.1001/archopht.1939.0086003 0092008

30. Winkelman JE. Central and peripheral fusion. AMA Arch Ophthalmol. 1953;50(2):179-183. doi:10.1001/archopht.1953.00920 030184006

31. Crone RA. Anomale Korrespondenz und anomales Binokularsehen [Abnormal correspondence and abnormal binocular vision]. Buch Augenarzt. 1978;72:49-62. German.

32. Sharples S, Cobb S, Moody A, Wilson JR. Virtual reality induced symptoms and effects (VRISE): comparison of head mounted display (HMD), desktop and projection display systems. Displays. 2008;29 (2):58-69
Clinical Ophthalmology

\section{Publish your work in this journal}

Clinical Ophthalmology is an international, peer-reviewed journal covering all subspecialties within ophthalmology. Key topics include: Optometry; Visual science; Pharmacology and drug therapy in eye diseases; Basic Sciences; Primary and Secondary eye care; Patient Safety and Quality of Care Improvements. This journal is indexed on PubMed

\section{Dovepress}

Central and CAS, and is the official journal of The Society of Clinical Ophthalmology (SCO). The manuscript management system is completely online and includes a very quick and fair peer-review system, which is all easy to use. Visit http://www.dovepress.com/ testimonials.php to read real quotes from published authors. 